\title{
Aspirin ameliorates cerebral infarction through regulation of TLR4/NF-кB-mediated endoplasmic reticulum stress in mouse model
}

\author{
XIN WANG* , BIN SHEN*, DEZHOU SUN and XIANGYU CUI \\ Department of Neurosurgery, Dezhou People's Hospital, Dezhou, Shandong 420065, P.R. China
}

Received November 30, 2016; Accepted August 14, 2017

DOI: $10.3892 / \mathrm{mmr} .2017 .7879$

\begin{abstract}
Cerebral infarction is a cerebrovascular disease caused by local brain ischemic necrosis or softening, which is associated with diabetes, obesity, hypertension and rheumatic heart arrhythmia. Previous studies have indicated that aspirin is a potential oral anticoagulant in the treatment of cerebral ischemic stroke. However, the potential mechanism mediated by aspirin in cerebral infarction therapy is not well understood. The present study analyzed the therapeutic effects of aspirin on cerebral infarction and investigated the underlying molecular mechanism of aspirin-ameliorated benefits for thrombolysis. The results demonstrated that aspirin inhibited inflammation and apoptosis of cerebrovascular endothelial cells in a mouse model of cerebral infarction. Aspirin treatment suppressed toll-like receptor (TLR) 4 and nuclear factor $(\mathrm{NF})-\kappa \mathrm{B}$ expression in cerebrovascular endothelial cells. Endoplasmic reticulum (ER) stress was suppressed by aspirin treatment through the downregulation of protein kinase R-like endoplasmic reticulum kinase, eukaryotic translation initiation factor 2 subunit 1 and $\mathrm{C} / \mathrm{EBP}$ homologous protein expression levels in cerebrovascular endothelial cells. It was identified that knockdown of TLR4 inhibited aspirin-mediated downregulation of $\mathrm{NF}-\kappa \mathrm{B}$ signaling pathway and ER stress in cerebrovascular endothelial cells. Expression levels of adenosine diphosphate plasminogen activator inhibitors, von Willebrand factor and thromboxane were downregulated in cerebrovascular endothelial cells and in serum in experimental mice. The results demonstrated that aspirin was beneficial forthrombolysis by decreasing thrombin-activatable fibrinolysis inhibitor and plasminogen activator inhibitor-1 expression in a mouse model of cerebral infarction. These
\end{abstract}

Correspondence to: Professor Xiangyu Cui, Department of Neurosurgery, Dezhou People's Hospital, 83 Fugui Road, Dezhou, Shandong 420065, P.R. China

E-mail: cuixiangyuedu@163.com

\section{*Contributed equally}

Key words: aspirin, cerebral infarction, toll-like receptor 4, anti-inflammation, nuclear factor- $\kappa \mathrm{B}$, endoplasmic reticulum stress results suggested that aspirin may improve cerebral infarction by downregulating TLR4/NF- $\kappa \mathrm{B}$-mediated ER stress in a mouse model.

\section{Introduction}

Cerebral infarction is a severe neurological disease that is characterized by obstacles to the brain blood supply or limitations in brain tissue and frequently leads to cerebral ischemic necrosis anoxic lesions $(1,2)$. Previous studies have suggested that cerebral infarction may result innerve dysfunction and is often associated with high blood pressure, diabetes, hyperlipidemia and other risk factors $(3,4)$. Clinical investigations indicate that pathological changes of blood vessel walls, blood composition changes and drug-induced or injury-caused cerebral artery dissection contribute to the initiation and development of cerebral infarction $(5,6)$. Thrombolytic treatments are the most commonly used therapy for patients suffering from cerebral infarction and it is necessary to understand the underlying therapeutic effects and potential molecular mechanisms in the progression of cerebral infarction.

The imbalance between coagulation and the fibrinolytic system serves an important role in the progression and pathogenesis of arterial thrombosis (7). Aspirin is effective and safe in the secondary prevention of cerebral infarction, as previously reported in a number of randomized, double-blind trials $(8,9)$. Tissue plasminogen activator (tPA) is an effective drug approved by US FDA for the treatment of ischemic stroke, and it exhibits pleiotropic effects in addition to thrombolysis (10). Thrombin-activatable fibrinolysis inhibitor (TAFI) is also an anti-fibrinolytic factor with an increased risk of cerebral infarction as the levels of plasma TAFI increase $(11,12)$. Previous studies have suggested that TAFI and plasminogen activator inhibitor (PAI)-1serve crucial roles in the initiation and development of deep cerebral infarction $(13,14)$. Inflammation and apoptosis of cerebrovascular endothelial cells increase the aggravation of cerebral infarction and other syndromes inpatients (14).

The present study investigated the therapeutic effects of aspirin on cerebral infarction in a mouse model. Potential molecular mechanisms mediated by aspirin were analyzed in cerebrovascular endothelial cells in a mouse model of cerebral infarction. The anti-inflammatory and anti-apoptotic effects 
of aspirin were also researched. The results indicated that aspirin can inhibit inflammation and apoptosis in addition to its profibrinolytic properties, as determined by in vitro and in vivo analysis. These findings suggested that aspirin may be a potential drug for cerebral infarction therapy, which may act by downregulating toll-like receptor (TLR)4/nuclear

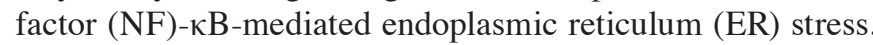

\section{Materials and methods}

Ethical approval and participant consent. The present preclinical study was approved by the Ethics Committee of Dezhou People's Hospital (Shandong, China). All surgeries and euthanasia of experimental animals were performed under sodium pentobarbital anesthesia to minimize pain.

Cells and reagents. Cerebrovascular endothelial cells were isolated from experimental mice according to a previous study (15) and cultured in minimum essential medium (Sigma-Aldrich; Merck KGaA, Darmstadt, Germany) supplemented with $10 \%$ fetal bovine serum (FBS; Gibco; Thermo Fisher Scientific, Inc., Waltham, MA, USA). Cells were cultured in a $37^{\circ} \mathrm{C}$ humidified atmosphere of $5 \% \mathrm{CO}_{2}$.

Reverse transcription-quantitative polymerase chain reaction $(R T-q P C R)$. Total RNA was extracted from cerebrovascular endothelial cells and serum using an RNA plus kit (Invitrogen; Thermo Fisher Scientific, Inc.) following the manufacturer's protocol, and treated with $2 \mathrm{U} / \mu \mathrm{g}$ DNase I (Invitrogen; Thermo Fisher Scientific, Inc.) at $37^{\circ} \mathrm{C}$ for $30 \mathrm{~min}$. A total of $5 \mu \mathrm{g}$ RNA per sample was primed with Oligo dT primer (Takara Biotechnology Co., Ltd., Dalian, China) and reverse transcribed using the AMV Reverse Transcription System (Invitrogen; Thermo Fisher Scientific, Inc.). For the PCR experiments, the following forward and reverse primers were used: Forward, 5'-TGGCAGCAGTGACAGCAGCA-3' and reverse, 5'-TACGGAGGTGGAGTGGGTGT-3' for ADP; forward, 5'-AGCCGAGGAAGAACTATGAAC-3' and reverse, 5'-ATTTGAGGGTGAGGAATGGG-3' for PAI-1; forward, 5'-GAGGGCAGAATCATCACGAAGT-3' and reverse, 5'-TGAGAGATCTGGTTCCCGAAAC-3' for vWF; forward, 5'-CAAGGCAGAGGTGGGTTTGG-3' and reverse, 5'-GGCACCTTTTCAGTTGCTCAC-3' for thromboxane; forward, 5'-CAA AGGTGGATCAGATTCAAG-3' and reverse, 5'-GGTGAGCATTATCACCCAGAA-3' for the reference gene GAPDH. The RT-qPCR mixture system comprised cDNA templates $(1 \mu \mathrm{l}$; Invitrogen; Thermo Fisher Scientific, Inc.), primers ( $2 \mu 1$ eachof the forward and reverse primers) and SYBR Green qPCR Master mix (5 $\mu$ l; Invitrogen; Thermo Fisher Scientific, Inc.). The PCR conditions included an initial denaturation step of $94^{\circ} \mathrm{C}$ for $2 \mathrm{~min}$, followed by 30 cycles of $94^{\circ} \mathrm{C}$ for $30 \mathrm{sec}, 59^{\circ} \mathrm{C}$ for $30 \mathrm{sec}, 72^{\circ} \mathrm{C}$ for $2 \mathrm{~min}$ and a final elongation step at $72^{\circ} \mathrm{C}$ for $10 \mathrm{~min}$. Taq DNA polymerase was purchased from Sigma-Aldrich; Merck KGaA. GAPDH was used as an internal control to normalize gene expression. The relative gene expression levels were calculated using the $2^{-\Delta \Delta \mathrm{Cq}}$ method (16). All experiments were repeated 3 times.

Western blot analysis. Cerebrovascular endothelial cells $\left(1 \times 10^{4}\right.$ cells $)$ were lysed using radioimmunoprecipitation assay lysis buffer (Invitrogen; Thermo Fisher Scientific, Inc.) and centrifuged $(1,000 \times \mathrm{g})$ at $4^{\circ} \mathrm{C}$ for $10 \mathrm{~min}$. The protein concentrations of the cell extracts were then measured using Bradford protein dye reagent (Bio-Rad Laboratories, Inc., Hercules, CA, USA). A total of $30 \mu \mathrm{g} /$ lane protein was loaded and separated by $12 \%$ SDS-PAGE and transferred to nitrocellulose membranes. The membranes were blocked with $5 \%$ skimmed milk for $1 \mathrm{~h}$ at room temperature, washed in Tris-buffered saline containing $0.1 \%$ Tween-20 (TBST) and incubated with the following primary antibodies: Anti-mouse IL-6 (1:2,000; cat no. ab7737; Abcam, Cambridge, UK); anti-mouse TNF- $\alpha$ (1:2,000; cat no. ab6671; Abcam); anti-mouse IL-1 $\beta$ (1:2,000; cat no. ab 9722; Abcam); anti-mouse caspase-3 (1:1,000; cat no. ab13847; Abcam); anti-mouse caspase-9 (1:1,000; cat. no. ab18571; Abcam); anti-mouse p53 (1:1,000; cat no. ab1431; Abcam); anti-mouse Bcl-2 (1:1,000; cat no. ab194583; Abcam); anti-mousecaspase-12 (1:1,000; cat. no. ab18766; Abcam); anti-mouse TLR-4 (1:1,000; cat no. ab13867; Abcam); anti-mouse p65 (1:1,000; cat. no. ab16502; Abcam); anti-mouse IKK $\beta$ (1:1,000; cat. no. ab53694; Abcam); anti-mouse $\operatorname{IkB} \alpha$ (1:1,000; cat. no. ab72429; Abcam); anti- protein kinase R-like endoplasmic reticulum kinase (PERK; 1:1,000; cat. no. ab65142; Abcam); anti-mouse eukaryotic translation initiation factor 2 subunit 1 (eIF2 $\alpha$; 1:1,000; cat. no. ab26197; Abcam); anti-mouse CHOP (1:1,000; cat. no. ab10444; Abcam); anti-mouseGRP78 (1:1,000; cat. no. ab32618; Abcam) and anti- $\beta$-actin $(1: 2,000$; cat. no. ab8226; Abcam) overnight at $4^{\circ} \mathrm{C}$. The labeled membranes were then washed three times with TBST, incubated for $2 \mathrm{~h}$ at room temperature with secondary anti-primary IgG conjugated with horseradish peroxidase (1:1,500; cat. no. ab6717; Abcam). The protein bands labeled with the antibodies were visualized using the SuperSignal West Pico Chemiluminescent Substrate Trial kit (Pierce Protein Biology; Thermo Fisher Scientific, Inc.). Images were obtained using the ChemiDoc XRS system with Quantity One software (Bio-Rad Laboratories, Inc.). Protein expression was analyzed using BandScan 5.0 software (Glyko, Inc., Novato, CA, USA). All experiments were repeated 3 times.

Animals. A total of 30 maleC57BL/6 mice (mean age of 6 months and a body weight of 20-25 g) were purchased from Vital River Laboratory Animal Technology Co. Ltd. (Shanghai, China). Mice were maintained in a room with constant temperature $\left(22 \pm 1^{\circ} \mathrm{C}\right)$ and a 12-h light/dark cycle, and cages in groups $<5$ per cage with ad libitum access to food and water. Mice were used to establish the model of cerebral infarction by local atherosclerosis cerebral ischemic necrosis according to previous study (17). Experimental mice were randomly divided into two groups after cerebral infarction surgery ( $\mathrm{n}=15$ in each group) and received aspirin or PBS treatment for 30 days. Aspirin (20 mg/kg body weight) or PBS was administered in drinking water once/day. The experimental mice were sacrificed under $1.5 \%$ pentobarbital sodium $(1 \mathrm{ml} / \mathrm{kg}$; Lianshuoinc, Shanghai, China) on day 30 for further analysis.

Immunohistochemical analysis. Cerebrovascular tissues were isolated from experimental mice following 30-day treatment with aspirin or PBS. The tissues in cerebral infarction were soaked in mixed stationary liquid (RongboBio, Shanghai, 
China) to fully fix for $24 \mathrm{~h}$ at room temperature. It was then washed in flowing water for $24 \mathrm{~h}$, and underwent conventional gradient alcohol dehydration, and xylene paraffin embedding. The wax blocks were cut into sections, with each section cut at $4 \mu \mathrm{m}$ thickness for immunohistochemical staining. The sections were dewaxed by conventional methods and underwent microwave antigen retrieval at $95^{\circ} \mathrm{C}$ for $10 \mathrm{~min}$. After cooling, they were washed with distilled water and blocked in normal fetal bovine serum (Gibco; Thermo Fisher Scientific, Inc.) at room temperature for $30 \mathrm{~min}$. The primary antibody TLR4 (1:500; cat no. ab13867; Abcam) and NF-кB (1:500; cat no. ab32360; Abcam) was added and placed at $4^{\circ} \mathrm{C}$ for $20 \mathrm{~h}$. The slides were allowed to equilibrate for $1 \mathrm{~h}$ at room temperature, and soaked in PBS four times for $5 \mathrm{~min}$. Then, secondary antibody (1:1,000; cat. no. ab6717; Abcam) was added and allowed to incubate at $37^{\circ} \mathrm{C}$ for $10 \mathrm{~min}$, after which the slides underwent PBS cleaning three times for $5 \mathrm{~min}$. Slides were observed with a BZ-9000 fluorescent video microscope (Keyence Corporation, Osaka, Japan). All experiments were repeated 3 times.

Assessment of thrombus formation in vivo. Thrombus formation in experimental mice was measured in using an Alexa Flour 488 (Invitrogen; Thermo Fisher Scientific, Inc.) ex vivo labeled anti-fibrin antibody (1:1,000; cat. no. ab34269; Abcam). Fluorescence intensity was quantified by intravital video microscopy (BX51WI; Olympus Corporation, Tokyo, Japan) using Image-Pro Plus (version 6.0, Media Cybernetics, Inc., Rockville, MD, USA).

ELISA. The expression of PERK (cat. no DEIA-XYA1959; Creative Diagnostics, USA), NF-KB (cat no. JK-(a)-6261; JingkangBioscience, Shanghai, China), and e eIF2 $\alpha$ (cat no. 7952S; Cell Signaling Technology, Inc., Danvers, MA, USA) in cerebrovascular endothelial cells were analyzed by ELISA kits and performed according to the manufacturer's protocols.

Knockdown of TLR4. TLR4 small interfering RNA (siRNA) and scramble siRNA (as a negative control) were purchased from Shanghai GenePharma Co., Ltd. (Shanghai, China). The sequences were as follows: si-TLR4, forward 5'-GCAUCU CUACAUUCAAGAA-3' and reverse 5'-UUCUUGAAUGUA GAGAUGC-3'; scrambled sequence (si-NC), forward 5'-UUC UCCGAACGUGUCACGU-3' and reverse 5'-ACGUGACAC GUUCGGAGAA-3'. For transfection, cerebrovascular endothelial cells at a density of $1 \times 10^{5}$ cells/well were seeded in each well of a 24-well microplate, grown for 24 h to reach $30-50 \%$ confluence and then incubated with TLR4 siRNA or scrambled siRNA and Lipofectamine ${ }^{\mathrm{TM}} 2000$ reagent (Invitrogen; Thermo Fisher Scientific, Inc.) in $100 \mu \mathrm{l}$ serum-free Dulbecco's Modified Eagle's medium (Gibco; Thermo Fisher Scientific, Inc.), according to the manufacturer's protocol. The CCN4 knockdown was confirmed by western blot analysis and RT-qPCR.

Apoptosis analysis. Cerebrovascular endothelial cells $\left(1 \times 10^{4}\right.$ cells) were isolated from experimental mice, trypsinized and collected by centrifugation $(1,500 \mathrm{xg})$ for $10 \mathrm{~min}$ in order to apoptosis analysis. Cell density was adjusted to
$5 \times 10^{6}$ cells $/ \mathrm{ml}$ with PBS, labeled with annexin V-fluorescein isothiocyanate (FITC) and propidium iodide (PI) using the Annexin V-FITC kit from BD Biosciences (Franklin Lakes, NJ, USA) and analyzed with a FACScan flow cytometer (BD Biosciences). Apoptotic rate of cerebrovascular endothelial cells was analyzed by flow cytometry (BD, Biosciences) using WinMDI software (version 2.9; BD Biosciences). All experiments were repeated 3 times.

Statistical analysis. All data were presented as mean \pm standard error of the mean. Statistical significance was established using two-tailed Student's t-test using SPSS software version 19.0 (IBM Corp., Armonk, NY, USA). P<0.05 was considered to indicate a statistically significant difference.

\section{Results}

Aspirin inhibits inflammatory cytokines in cerebrovascular endothelial cells and serum in a mouse model of cerebral infarction. Inflammation has been regarded as a therapeutic target in cerebral infarction (17). The present study analyzed the cellular inflammatory response and the levels of inflammatory mediators in cerebrovascular endothelial cells and serum in mice with cerebral infarction. Serum concentrations of interleukin (IL)-6, tumor necrosis factor (TNF)- $\alpha$ and IL-1 $\beta$ were significantly decreased in aspirin-treated cerebral infarction mice compared with expression levels in control mice (Fig. 1A-C, respectively). Western blot analysis demonstrated that protein expression levels of IL- 6 , TNF- $\alpha$ and IL-1 $\beta$ were downregulated in cerebrovascular endothelial cells following treatment with aspirin (Fig. 1D-F, respectively). These results suggested that aspirin may inhibit inflammatory cytokine expression in cerebrovascular endothelial cells and serum in a mouse model of cerebral infarction.

Aspirin inhibits apoptosis of cerebrovascular endothelial cells in a mouse model of cerebral infarction. The anti-apoptotic effects of aspirin in a cerebral infarction mouse model were evaluated. The rate of apoptosis of cerebrovascular endothelial cells was significantly decreased by aspirin treatment, compared with control cells (Fig. 2A). Results from western blot analysis demonstrated that caspase-12, caspase- 3 and caspase- 9 expression levels were downregulated in aspirin-treated mice compared with control mice (Fig. 2B-D, respectively). However, expression levels of antiapoptoticp53 and B-cell lymphoma 2(Bcl-2) proteins were significantly increased in cerebrovascular endothelial cells in the aspirin-treated mice compared with the controls (Fig. 2E and F). These results indicated that aspirin may inhibit apoptosis in cerebrovascular endothelial cells in a mouse model of cerebral infarction.

Aspirin treatment suppresses TLR4, NF- $\kappa B, p 65, I \kappa B \alpha, I K K \beta$, ADP, PAI-1, VWF and thromboxane expression in cerebrovascular endothelial cells. To explore the therapeutic effects of aspirin, TLR4 and NF- $\mathrm{B}$ expression in cerebrovascular endothelial cells was analyzed. The protein expression level of TLR4 was downregulated in cerebrovascular endothelial cells in aspirin-treated mice, compared with control mice (Fig. 3A). The results also demonstrated that aspirin treatment reduced the protein expression levels of NF- $\mathrm{kB}$ p65, inhibitor of 
A

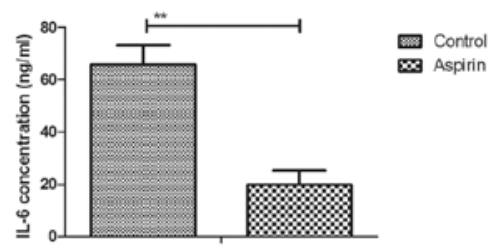

D

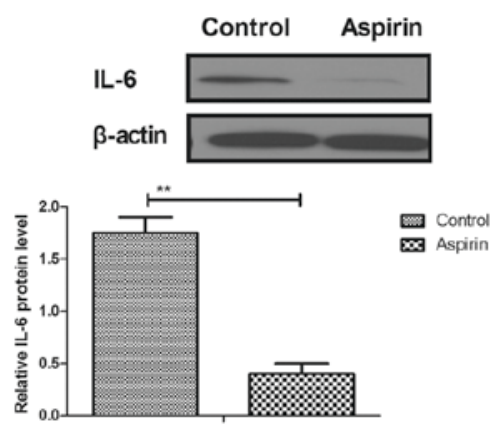

B

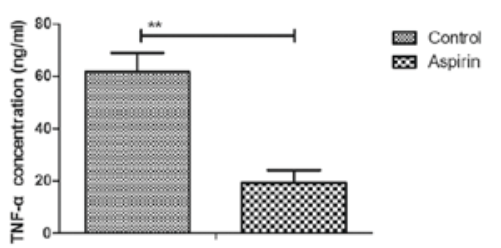

E

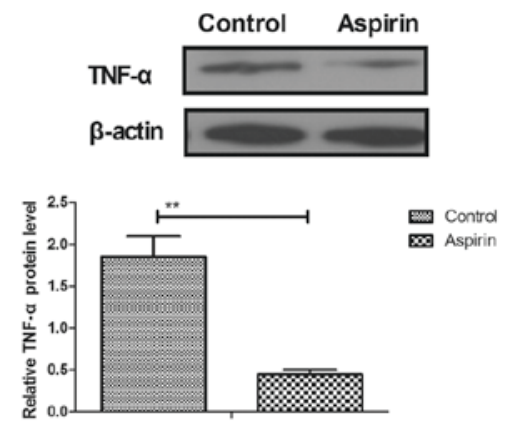

C

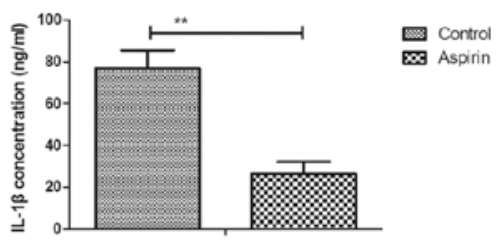

$\mathbf{F}$
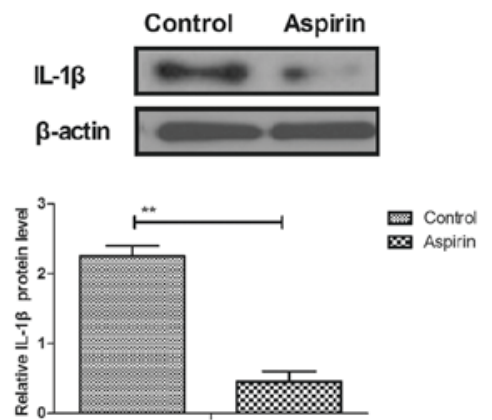

Figure 1. Effects of aspirin on inflammatory cytokines in serum and cerebrovascular endothelial cells in a mouse model of cerebral infarction. (A-C) Serum concentration levels of (A) IL-6, (B) TNF- $\alpha$ and (C) IL-1 $\beta$ in cerebrovascular endothelial cells in a mouse model of cerebral infarction treated by aspirin. (D-E) Aspirin treatment significantly reduced the protein expression levels of (D) IL-6, (E) TNF- $\alpha$ and (F) IL-1 $\beta$ in cerebrovascular endothelial cells in a mouse model of cerebral infarction. IL, interleukin; TNF, tumor necrosis factor. ${ }^{* *} \mathrm{P}<0.01$ vs. control group.

A
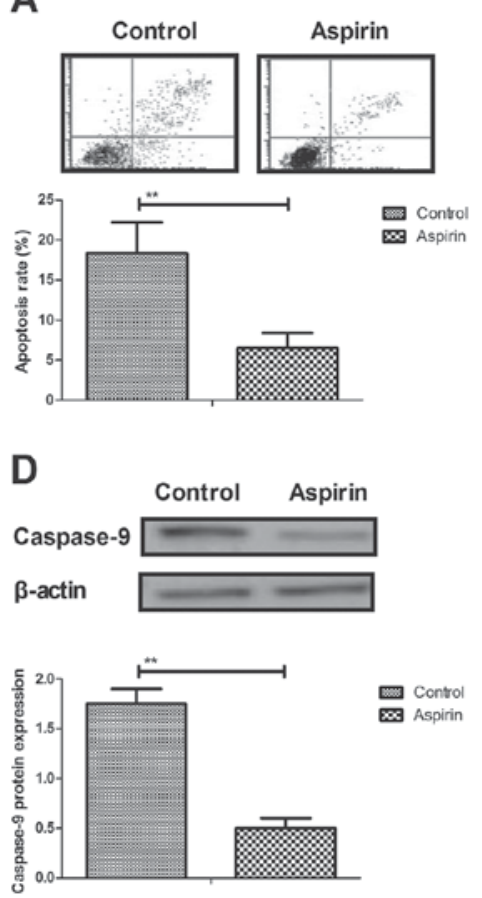

B

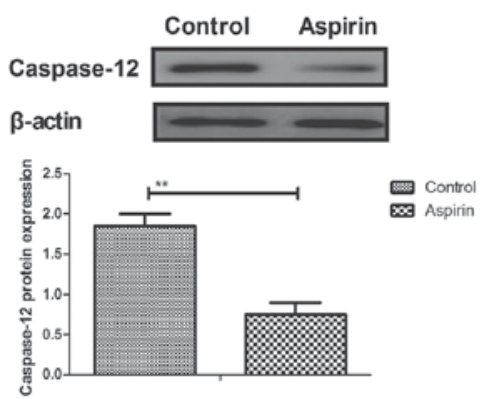

E
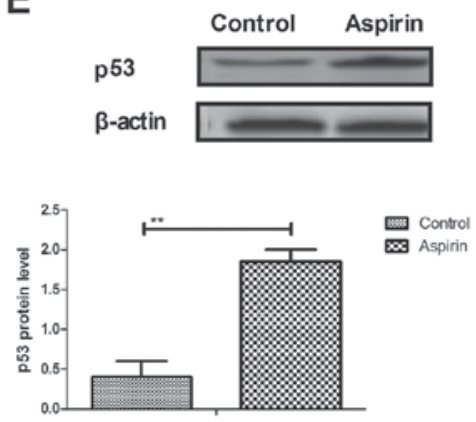

C

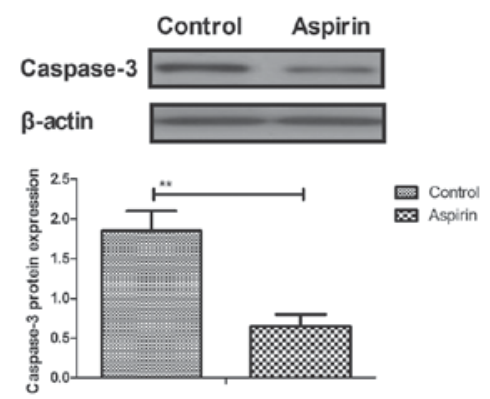

$\mathbf{F}$
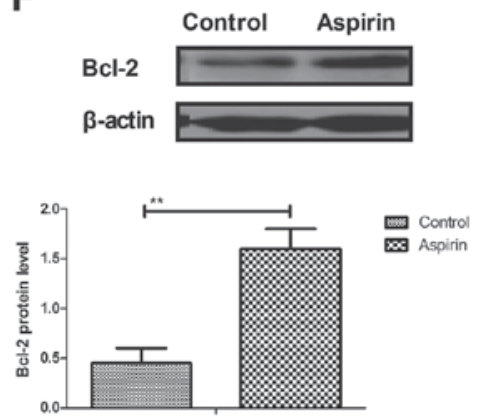

Figure 2. Aspirin treatment inhibits apoptosis in cerebrovascular endothelial cells in a mouse model of cerebral infarction. (A) Effects of aspirin treatment on apoptosis in cerebrovascular endothelial cells in a mouse model of cerebral infarction as measured by flow cytometry. Cell apoptosis was detected by flow cytometry. The cells were assessed by flow cytometry following Annexin V staining. Q4-2, early apoptotic cells positively stained for Annexin V-fluorescein isothiocyanate (FITC), and negative for propidium iodide (PI); Q3-2, normal cells not stained by Annexin V-FITC or PI; Q2-2, necrotic cells and late apoptotic cells stained by both Annexin V-FITC and PI. (B-D) Effects of aspirin treatment on the expression levels of (B) caspase-12, (C) caspase-3 and (D) caspase-9 in cerebrovascular endothelial cells. (E and F) Effects of aspirin on the protein expression levels of antiapoptotic (E) p53 and (F) Bcl-2. Bcl-2, B-cell lymphoma 2. ${ }^{* *} \mathrm{P}<0.01$ vs. control group.

NF- $\kappa B$ kinase (IKK) $\beta$ and NF- $\kappa B$ inhibitor (I $\mathrm{KB}) \alpha$ in cerebrovascular endothelial cells compared with control-treated cells (Fig. 3B-D, respectively). RT-qPCR analysis revealed that the
mRNA expression levels of activator inhibitor type-1 (ADP), (PAI-1), von Willebrand factor (VWF) and thromboxane were significantly reduced in cerebrovascular endothelial cells 
A

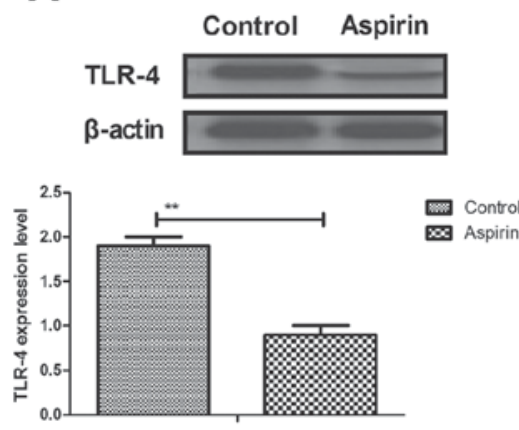

D
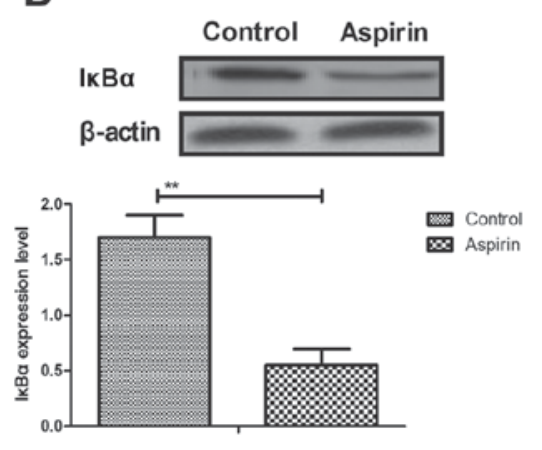

B
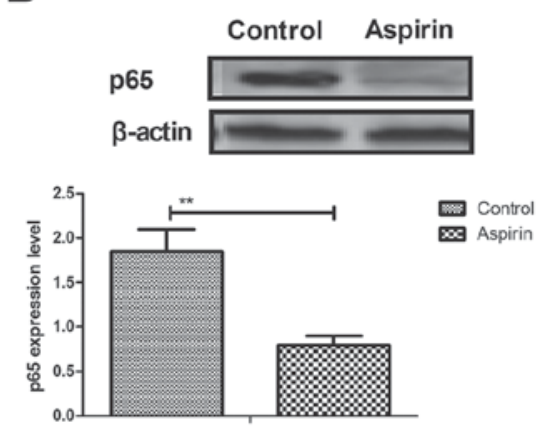

E

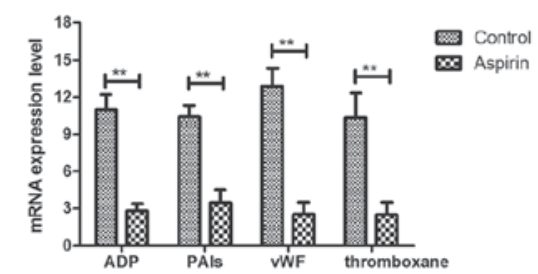

C
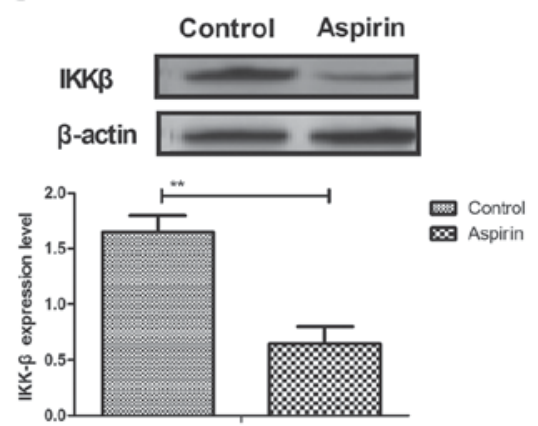

$\mathbf{F}$

Figure 3. Aspirin inhibits TLR4 and NF-kB expression in cerebrovascular endothelial cells. (A) Aspirin inhibited TLR4 expression levels in cerebrovascular

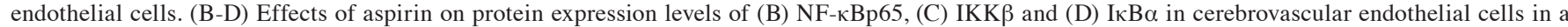
mouse model of cerebral infarction. (E) mRNA expression and (F) serum concentration levels of ADP, PAIs, vWF and thromboxanein experimental mice.

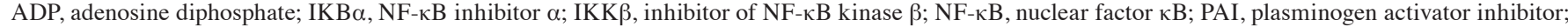
TLR, toll-like receptor; vWF, von Willebrand factor. ${ }^{* *} \mathrm{P}<0.01$ vs. control group.

A
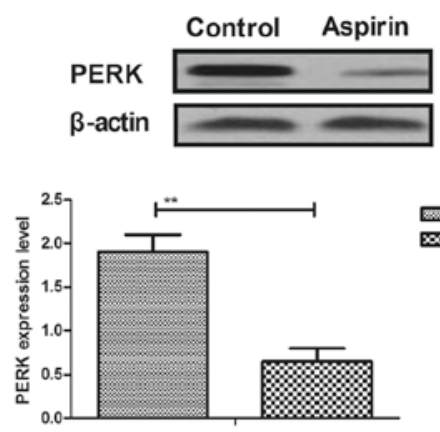

D
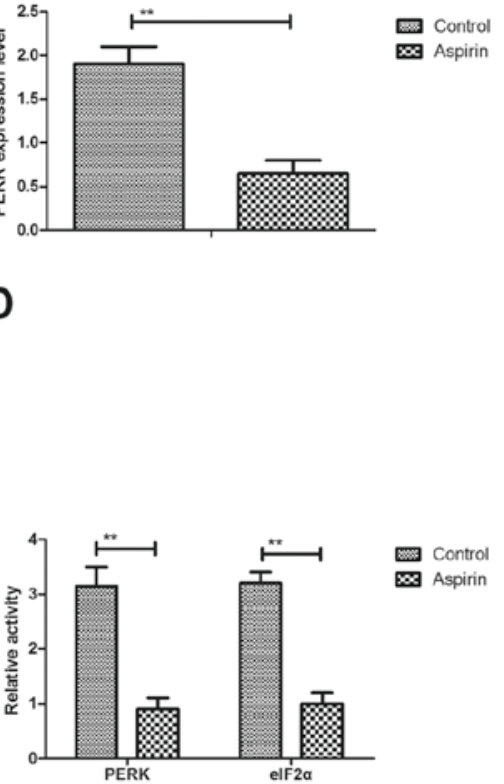

B
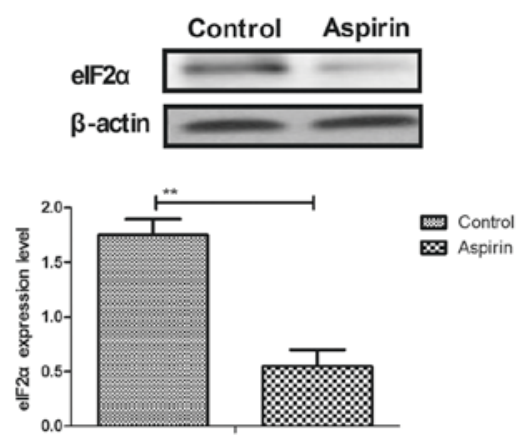

E

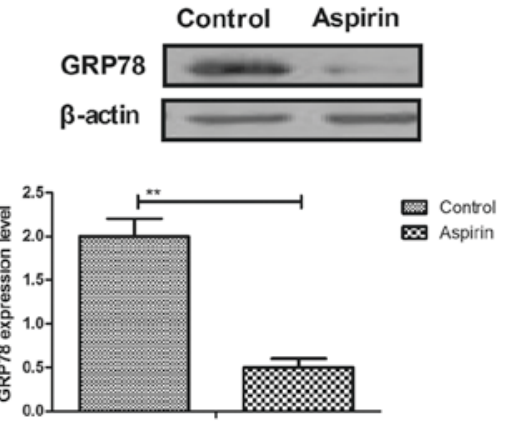

C
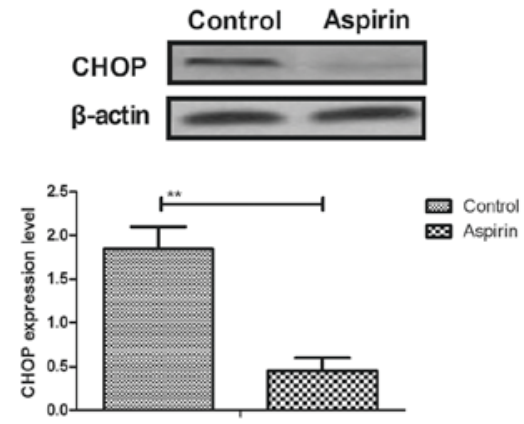

$\mathbf{F}$
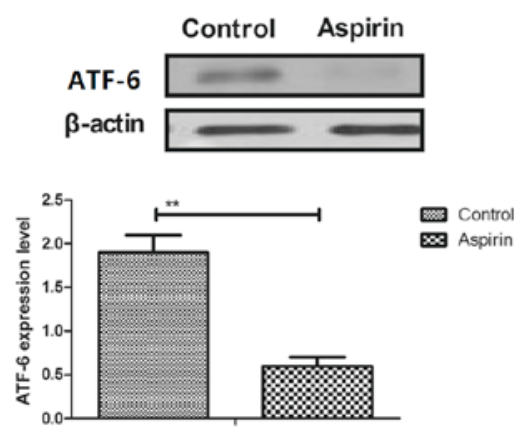

Figure 4. Knockdown of CCN4 inhibits the proliferation of cerebrovascular endothelial cells under hypoxic conditions. Aspirin inhibited the protein expression levels of (A) PERK, (B) eIF2 $\alpha$ and (C) CHOP. (D) The relative activities of PEAK and eIF2 $\alpha$, (E) GPR78 and (F) ATF6 in cerebrovascular endothelial cells in a mouse model of cerebral infarction. ATF, activating transcription factor; CHOP, C/EBP homologous protein; eIF2 $\alpha$, eukaryotic translation initiation factor 2 subunit 1; GRP, glucose-regulated protein; PERK, protein kinase R-like endoplasmic reticulum kinase.* $\mathrm{P}<0.01$ vs. control group. 
A

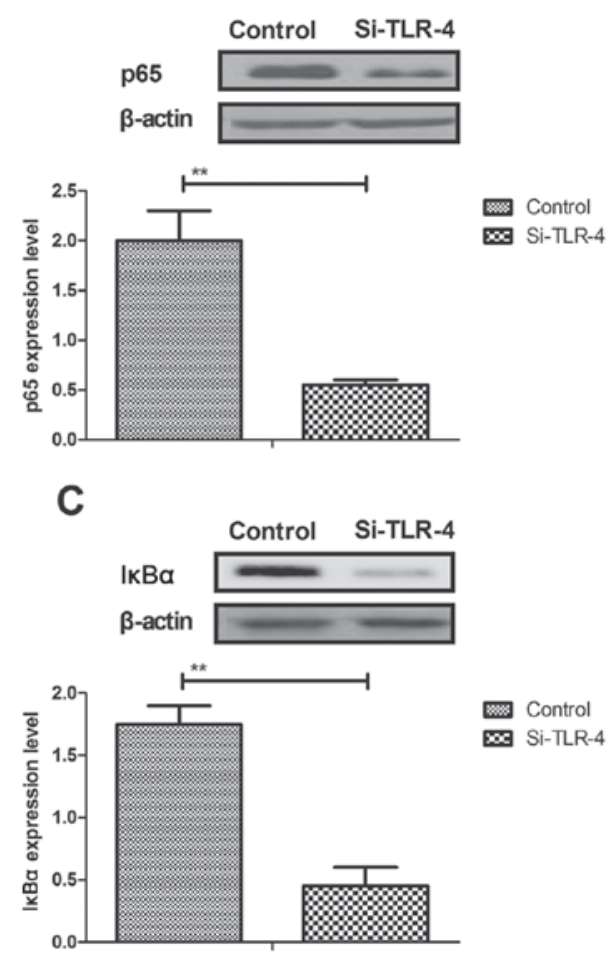

E
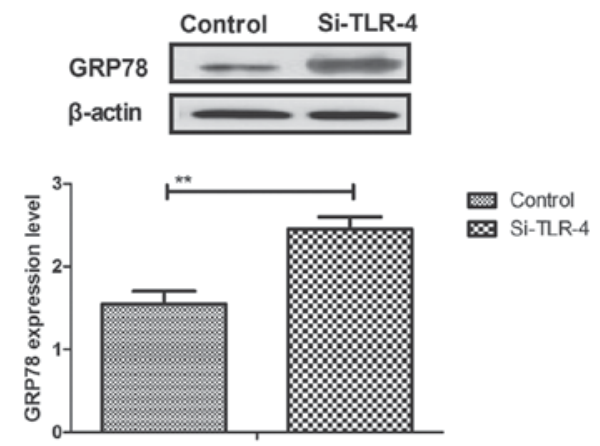

G
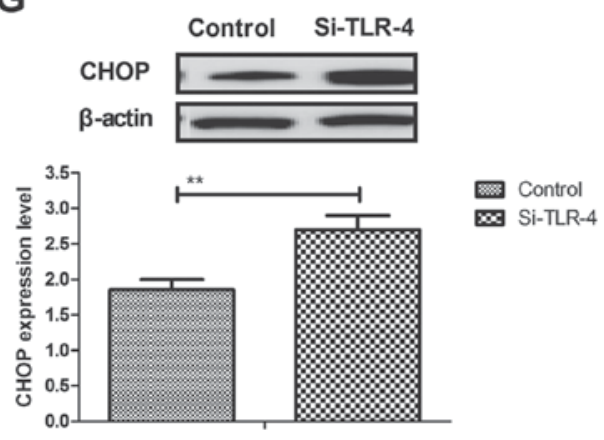

B

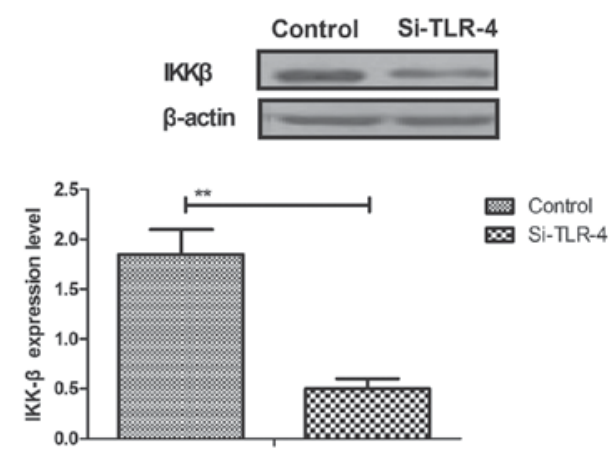

D

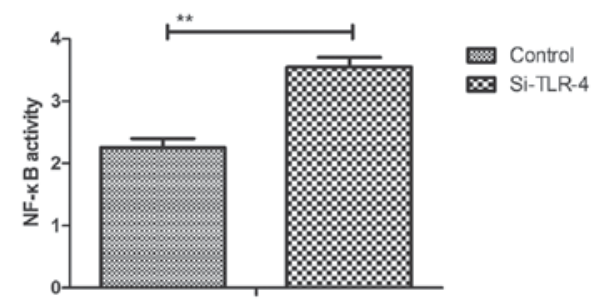

$\mathbf{F}$
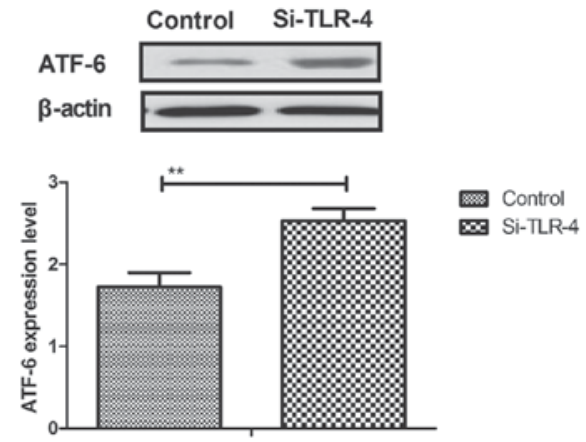

H

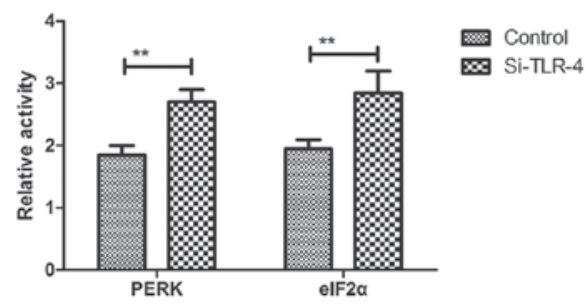

Figure 5. Aspirin regulates ER stress through the TLR4/NF- $\mathrm{kB}$ signaling pathway in cerebrovascular endothelial cells. (A and B) Knockdown of TLR4

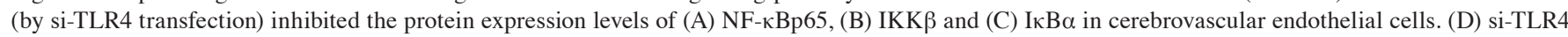
transfected cells also exhibited downregulated NF- $\mathrm{kB}$ activity in cerebrovascular endothelial cells. (E and G) si-TLR4 treatment increased the aspirin-inhibited expression levels of (E) GRP78, (F) ATF-6 and (G) CHOP in cerebrovascular endothelial cells. (H) si-TLR4 reversed the aspirin-induced inhibition of PERK and eIF2 $\alpha$ activity in cerebrovascular endothelial cells. ATF, activating transcription factor; CHOP, C/EBP homologous protein; eIF2 $\alpha$, eukaryotic translation

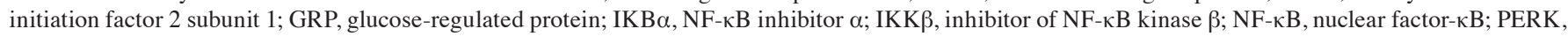
protein kinase R-like endoplasmic reticulum kinase; si, small interfering RNA; TLR, toll-like receptor. ${ }^{* *} \mathrm{P}<0.01$ vs. control group.

(Fig. 3E) and in serum (Fig. 3F) in experimental mice. These results demonstrated that aspirin treatment may suppress
TLR4NF-кB, p65, ADP, PAI, VWF and thromboxane expression in cerebrovascular endothelial cells. 
A

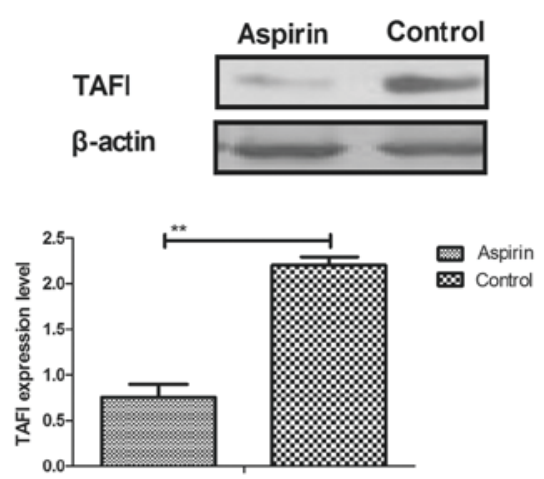

D

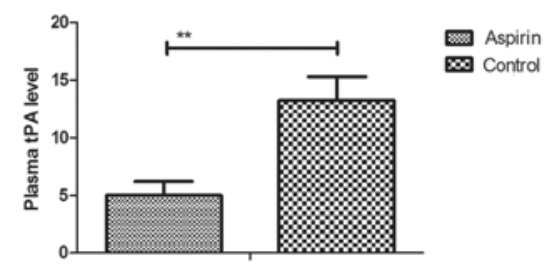

B
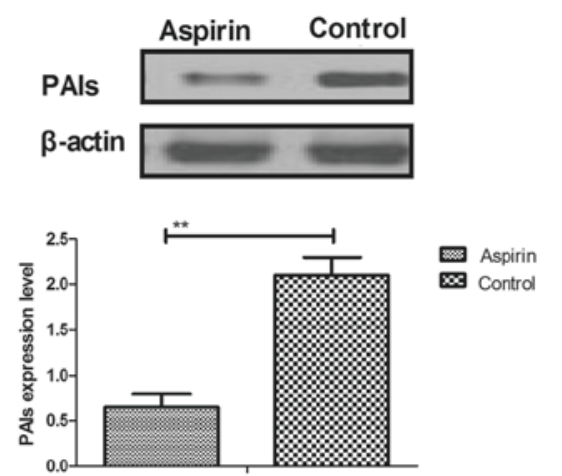

E

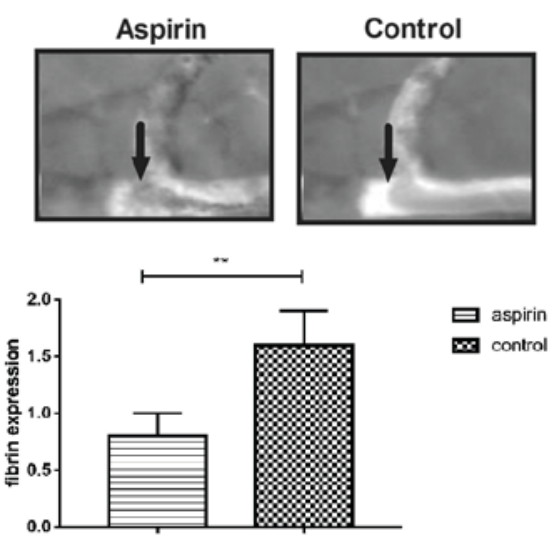

C

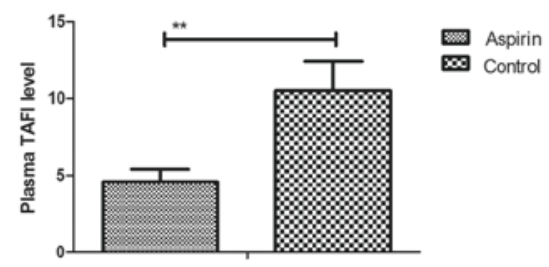

F

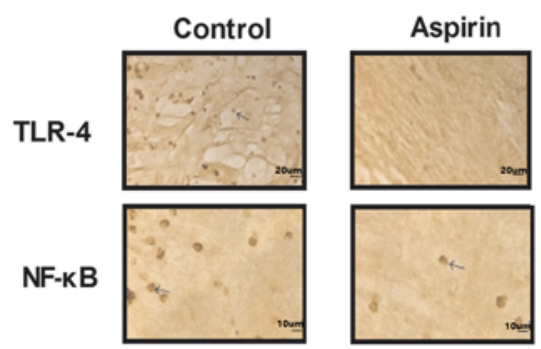

Figure 6. In vivo therapeutic effects of aspirin in a mouse model of cerebral infarction. (A and B) Aspirin treatment increased the expression levels of (A) TAFI and (B) PAIs in cerebrovascular endothelial cells. Effects of aspirin treatment on plasma concentration levels of (C) TAFI and (D) tPA in a mouse model of cerebral infarction. (E) Aspirin suppress thrombolysis in a mouse model of cerebral infarction (x400). (F) Aspirin downregulated TLR4 and NF- $\kappa \mathrm{B}$ expression levels as determined by immunohistochemistry. NF- $\mathrm{B}$, nuclear factor- $\mathrm{B}$; PAI, plasminogen activator inhibitor; TAFI, thrombin-activatable fibrinolysis inhibitor; TLR, toll-like receptor; tPA, tissue plasminogen activator. ${ }^{* *} \mathrm{P}<0.01$ vs. control group.

Aspirin treatment decreases ER stress in cerebrovascular endothelial cells in a mouse model of cerebral infarction. Changes in ER stress in cerebrovascular endothelial cells in a mouse model of cerebral infarction were investigated. As demonstrated in Fig. 4A-C, the protein expression levels of PERK, eIF2 $\alpha$ and C/EBP homologous protein (CHOP) were significantly downregulated by aspirin treatment in cerebrovascular endothelial cells, compared with expression levels in control cells. The data also indicated that PERK and eIF2 $\alpha$ activity were downregulated by aspirin in cerebrovascular endothelial cells (Fig. 4D). In addition, the protein expression levels of glucose-regulated protein (GRP)78 and activating transcription factor (ATF)-6 were significantly downregulated by aspirin treatment in cerebrovascular endothelial cells (Fig. 4E and F, respectively). These results suggest that aspirin treatment decreases ER stress in cerebrovascular endothelial cells in a mouse model of cerebral infarction.

Aspirin treatment regulates ER stress through TLR4-NF- $\mathrm{TB}$ signaling pathway in cerebrovascular endothelial cells. The potential mechanism of aspirin-mediated improvements of ER stress was investigated. The results demonstrated that knockdown of TLR4by si-TLR4 transfection inhibited the protein expression levels of p65, IKK $\beta$ and I $\mathrm{B} \alpha \alpha$ (Fig. 5A-C, respectively), and increased $\mathrm{NF}-\kappa \mathrm{B}$ activity in cerebrovascular endothelial cells (Fig. 5D). Aspirin-inhibited expression levels of GRP78, ATF-6 and CHOP were canceled by TLR4 knockdown in cerebrovascular endothelial cells (Fig. 5E-G). TLR4 knockdown also inhibited aspirin-downregulated PERK and eIF2 $\alpha$ activity in cerebrovascular endothelial cells (Fig. 5H). These results demonstrated that aspirin treatment downregulated ER stress through the TLR4-NF- $\kappa \mathrm{B}$ signaling pathway in cerebrovascular endothelial cells.

In vivo efficacy of aspirin for a mouse model of cerebral infarction. The present study further analyzed the in vivo efficacy of aspirin for cerebral infarction in a mouse model. The results demonstrated that aspirin treatment may suppress thrombolysis through increasing expression levels of TAFI and PAI-1 in cerebrovascular endothelial cells (Fig. 6A and B, respectively). TAFI and tPA plasma concentration levels were significantly decreased in cerebral infarction model mice following treatment with aspirin, compared with control-treated mice (Fig. 6C and D, respectively). The result revealed that aspirin could suppress thrombolysis in a mouse model of cerebral infarction (Fig. 6E). Immunohistochemical analysis demonstrated that TLR4 and NF- $\kappa \mathrm{B}$ expression levels were downregulated by aspirin treatment in cerebrovascular 
lesions (Fig. 6F). These results suggested that aspirin may be beneficial for the treatment of cerebral infarction.

\section{Discussion}

Cerebral infarction is a combination of pathophysiological processes that are induced by local atherosclerosis cerebral ischemic necrosis $(18,19)$. Thrombolytic treatments for cerebral infarction are efficient in clearing cerebrovascular congestion in patients $(5,20)$. The therapeutic effects of aspirin for cerebral infarction by anti-platelet aggregation have been investigated in previous studies $(21,22)$. The present study investigated the potential mechanism of aspirin-mediated treatments of cerebral infarction in an animal model. The present study indicated that aspirin inhibits inflammation and apoptosis of cerebrovascular endothelial cells in experimental mice induced with autologous arterial blood clot. The results also indicated that aspirin treatment may inhibit ER stress through the suppression of TLR4-mediated NF- $\mathrm{BB}$ signaling in cerebrovascular endothelial cells, which may contribute to thrombolysis in a mouse model of cerebral infarction

Inflammation is associated with the progression of cerebral infarction. A previous study demonstrated that plasma concentration levels of IL-6, TNF- $\alpha$ and IL- $1 \beta$ were upregulated in patients with cerebral infarction $(17,23,24)$. Another study revealed that the inflammation-related NF- $\kappa B$ signaling pathway was activated in patients with acute cerebral infarction (25). It has also been indicated that cytokine production and NF- $\mathrm{KB}$ activation were upregulated in peripheral blood mononuclear cells of patients with cerebral infarction (26). In the present study, the data indicated that aspirin treatment downregulated inflammation and NF- $\mathrm{kB}$ signaling in cerebrovascular endothelial cells in a mouse model of cerebral infarction.

It has been reported that apoptosis and ER stress are associated with aggravation of cerebrovascular diseases $(27,28)$. Increased ER stress in cerebrovascular endothelial cells contributes to apoptosis of cells and aggravation of cerebral infarction (29). Chen et al (30) demonstrated that the TLR4/NF- $\mathrm{kB}$ pathway was involved in cognitive impairment, and neuro-inflammatory and apoptotic responses. The results of the present study demonstrated that aspirin treatment downregulated TLR4 and NF- $\mathrm{BB}$ expression, which further decreased ER stress in cerebrovascular endothelial cells in a mouse model of cerebral infarction.

The present study investigated the efficacy of aspirin treatment in vivo and demonstrated that aspirin treatment improved thrombolysis by increasing the expression levels of TAFI and PAI-1 in cerebrovascular endothelial cells. One previous study provided further insight into the mechanism of activated TAFI self-destruction, which indicated that TAFI deletion mutant appears to be more stable than the activated TAFI control (31). The tPA plasma concentration levels were increased in the mouse model of cerebral infarction following treatment with aspirin in the present study. In addition, previous studies have also has indicated the PAI-1regulates the balance of the plasma fibrinolytic and the blood coagulation systems, and further initiates or promotes the progression of cardiovascular disease $(32,33)$. The findings of the present study have suggested that aspirin promoted thrombolysis in a mouse model of cerebral infarction through decreasing TLR4 and NF- $\mathrm{\kappa B}$ expression levels in cerebrovascular lesions.

In conclusion, the present study examined the therapeutic effects and potential mechanisms of aspirin in the treatment of cerebral infarction. The results suggested that in a mouse model of cerebral infarction aspirin treatment improved cerebral infarction through decreasing inflammation and ER stress in cerebrovascular endothelial cells and may have also promoted thrombolysis through increasing the expression levels of ADP, PAIs, VWF and thromboxane. Compared with previous studies, the present study suggested that aspirin may modify cerebral infarction by decreasing TLR4 and NF- $\kappa \mathrm{B}$ expression levels via mediated ER stress in a mouse model. This suggested that aspirin may contribute to thrombolysis through the regulation ofTLR4/NF-kB-mediated ER stress in mice model.

\section{References}

1. Serramito García R, Santín Amo JM, Román Pena P, Pita Buezas L, González Gómez L and García Allut A: Cerebral infarction after pituitary apoplexy: Description of a case and review of the literature. Neurocirugia (Astur) 27: 310-314, 2016 (In Spanish).

2. Romi F and Naess H: Spinal cord infarction in clinical neurology: A review of characteristics and long-term prognosis in comparison to cerebral infarction. Eur Neurol 76: 95-98, 2016.

3. Arch AE and Sheth KN: Malignant cerebral edema after large anterior circulation infarction: A review. Curr Treat Options Cardiovasc Med 16: 275, 2014.

4. Russek NS and Jensen MB: Histological quantification of brain tissue inflammatory cell infiltration after focal cerebral infarction: A systematic review. Int J Neurosci 124: 160-165, 2014.

5. Hagiwara S, Yoshida A, Omata Y, Tsukada Y, Takahashi H, Kamewada H, Koike S, Okuzumi K, Hishinuma A, Kobayashi K and Nakano M: Desulfovibrio desulfuricans bacteremia in a patient hospitalized with acute cerebral infarction: Case report and review. J Infect Chemother 20: 274-277, 2014.

6. Chiba F, Makino Y, Motomura A, Inokuchi G, Ishii N, Torimitsu S, Sakuma A, Nagasawa S, Saito H, Yajima D, et al: Bilateral middle cerebral artery infarction associated with traumatic common carotid artery dissection: A case report and review of literature. Forensic Sci Int 236: e1-e4, 2014.

7. Shi J, Zhi P, Chen J, Wu P and Tan S: Genetic variations in the thrombin-activatable fibrinolysis inhibitor gene and risk of cardiovascular disease: A systematic review and meta-analysis. Thromb Res 134: 610-616, 2014.

8. Shinohara Y, Nishimaru K, Sawada T, Terashi A, Handa S, Hirai S, Hayashi K, Tohgi H, Fukuuchi Y, Uchiyama S, et al: Sarpogrelate-aspirin comparative clinical study for efficacy and safety in secondary prevention of cerebral infarction (S-ACCESS): A randomized, double-blind, aspirin-controlled trial. Stroke 39: 1827-1833, 2008.

9. Li YS: Aspirin in secondary prevention of cerebral infarction. Zhonghua Nei Ke Za Zhi 46: 625-627, 2007 (In Chinese).

10. Dong MX, Hu QC, Shen P, Pan JX, Wei YD, Liu YY, Ren YF, Liang ZH, Wang HY, Zhao LB and Xie P: Recombinant tissue plasminogen activator induces neurological side effects independent on thrombolysis in mechanical Animal models of focal cerebral infarction: A systematic review and meta-analysis. PloS One 11: e0158848, 2016.

11. Dubis J, Zuk N, Grendziak R, Zapotoczny N, Pfanhauser M and Witkiewicz W: Activity of thrombin-activatable fibrinolysis inhibitor in the plasma of patients with abdominal aortic aneurysm. Blood Coagul Fibrinolysis 25: 226-231, 2014.

12. Naderi M, Dorgalaleh A, Alizadeh S, Kashani Khatib Z, Tabibian S, Kazemi A, Dargahi H and Bamedi T: Polymorphism of thrombin-activatable fibrinolysis inhibitor and risk of intracranial haemorrhage in factor XIII deficiency. Haemophilia 20: e89-e92, 2014.

13. Yaroglu Kazanci S, Yesilbas O, Ersoy M, Kihtir HS, Yildirim HM and Sevketoglu E: Cerebral infarction and femoral venous thrombosis detected in a patient with diabetic ketoacidosis and heterozygous factor V Leiden G1691A and PAI-1 4G/5G mutations. J Pediatr Endocrinol Metab 28: 1183-1186, 2015. 
14. Akatsu H, Yamagata H, Chen Y, Miki T, Kamino K, Takeda M, Campbell W, Kondo I, Kosaka K, Yamamoto T and Okada H: TAFI polymorphisms at amino acids 147 and 325 are not risk factors for cerebral infarction. Br J Haematol 127: 440-447, 2004

15. Wei YP, Kita M, Shinmura K, Yan XQ, Fukuyama R, Fushiki S and Imanishi J: Expression of IFN-gamma in cerebrovascular endothelial cells from aged mice. J Interferon Cytokine Res 20: 403-409, 2000

16. Livak KJ and Schmittgen TD: Analysis of relative gene expression data using real-time quantitative PCR and the $2^{-\Delta \Delta C T}$ method. Methods 25: 402-408, 2001.

17. Cuenca-López MD, Brea D, Segura T, Galindo MF, Antón-Martínez D, Agulla J, Castillo J and Jordán J: Inflammation as a therapeutic agent in cerebral infarction: cellular inflammatory response and inflammatory mediators. Rev Neurol 50: 349-359, 2010 (In Spanish).

18. Yan $\mathrm{Z}$, Yu T, Wang $\mathrm{Y}$, Wang $\mathrm{M}$ and Liang $\mathrm{H}$ : Literature review and case report of intravenous thrombolysis in acute cerebral infarction attributed to cervical arterial dissection. J Stroke Cerebrovasc Dis 24: e265-e269, 2015.

19. Taylor B, Lopresti M, Appelboom G and Sander Connolly E Jr: Hemicraniectomy for malignant middle cerebral artery territory infarction: An updated review. J Neurosurg Sci 59: 73-78, 2015.

20. Sha D, Fan G and Zhang J: Multiple cerebral infarction as the initial manifestation of left atrial myxoma: A case report and literature review. Acta Cardiol 69: 189-192, 2014.

21. Cohen LK and Jensen MB: Scaffolds for intracerebral grafting of neural progenitor cells after cerebral infarction: A systematic review. Arch Neurosci 2: e25364, 2015.

22. Zhang C, Feng F, Zhu Y, Wang R and Xing B: Cerebral infarction caused by pituitary apoplexy: Case report and review of literature. Turk Neurosurg 24: 782-787, 2014.

23. Shimamura N, Matsuda N, Kakuta K, Narita A and Ohkuma H: A model of rat embolic cerebral infarction with a quantifiable, autologous arterial blood clot. Transl Stroke Res 4: 564-570, 2013.

24. Fang MF, Tan F and Zhang X: Effects of Huatan Tongluo Granule on SOCS-3 and TNF- $\alpha$ expressions in patients with acute cerebral infarction. Zhongguo Zhong Xi Yi Jie He Za Zhi 30: 1142-1145, 2010 (In Chinese).

25. Jiang Y and Lian YJ: Effects of Danhong injection on hemodynamics and the inflammation-related NF- $\kappa$ B signaling pathway in patients with acute cerebral infarction. Genet Mol Res 14: $16929-16937,2015$.
26. Kim SJ, Jeong HJ, Lee KM, Moon PD, Yun JM, Cho KH, Moon BS, Lee HJ, Hong SH, Kim HM and Um JY: The effect of SHJKS on cytokines production and $\mathrm{NF}-\kappa \mathrm{B}$ activation in the peripheral blood mononuclear cells of patients with cerebral infarction. Immunopharmacol Immunotoxicol 28: 557-570, 2006.

27. Li M, Peng J, Wang MD, Song YL, Mei YW and Fang Y: Passive movement improves the learning and memory function of rats with cerebral infarction by inhibiting neuron cell apoptosis. Mol Neurobiol 49: 216-221, 2014.

28. Li B, Tian J, Sun Y, Xu TR, Chi RF, Zhang XL, Hu XL, Zhang YA, Qin FZ and Zhang WF: Activation of NADPH oxidase mediates increased endoplasmic reticulum stress and left ventricular remodeling after myocardial infarction in rabbits. Biochim Biophys Acta 1852: 805-815, 2015.

29. Li M, Peng J, Song Y, Liang H, Mei Y and Fang Y: Electro-acupuncture combined with transcranial magnetic stimulation improves learning and memory function of rats with cerebral infarction by inhibiting neuron cell apoptosis. J Huazhong Univ Sci Technolog Med Sci 32: 746-749, 2012.

30. Chen L, Hu L, Zhao J, Hong H, Feng F, Qu W and Liu W: Chotosan improves A $\beta 1$-42-induced cognitive impairment and neuroinflammatory and apoptotic responses through the inhibition of TLR-4/NF- $\mathrm{BB}$ signaling in mice. J Ethnopharmacol 191: 398-407, 2016.

31. Plug T and Meijers JC: New clues regarding the mysterious mechanism of activated thrombin-activatable fibrinolysis inhibitor self-destruction. J Thromb Haemost 13: 1081-1083, 2015.

32. Oztuzcu S, Ergun S, Ulaşlı M, Nacarkahya G, Iğci YZ, Iğci M, Bayraktar R, Tamer A, Çakmak EA and Arslan A: Evaluation of factor V G1691A, prothrombin G20210A, factor XIII V34L, MTHFR A1298C, MTHFR C677T and PAI-1 4G/5G genotype frequencies of patients subjected to cardiovascular disease (CVD) panel in south-east region of Turkey. Mol Biol Rep 41: 3671-3676, 2014

33. Hilbers FS, Boekel NB, van den Broek AJ, van Hien R, Cornelissen S, Aleman BM, van't Veer LJ, van Leeuwen FE and Schmidt MK: Genetic variants in TGF $\beta-1$ and PAI-1 as possible risk factors for cardiovascular disease after radiotherapy for breast cancer. Radiother Oncol 102: 115-121, 2012. 